ANNALES

POLONICI MATHEMATICI

$83.2(2004)$

\title{
A note on Costara's paper
}

\author{
by Armen Edigarian (Kraków)
}

Abstract. We show that the symmetrized bidisc $\mathbb{G}_{2}=\left\{\left(\lambda_{1}+\lambda_{2}, \lambda_{1} \lambda_{2}\right):\left|\lambda_{1}\right|,\left|\lambda_{2}\right|\right.$ $<1\} \subset \mathbb{C}^{2}$ cannot be exhausted by domains biholomorphic to convex domains.

Let $\mathbb{D}$ be the unit disc in $\mathbb{C}$. The open symmetrized bidisc $\mathbb{G}_{2}$ is the image of the bidisc $\mathbb{D}^{2}$ under the "symmetrization map" $\pi:\left(\lambda_{1}, \lambda_{2}\right) \mapsto$ $\left(\lambda_{1}+\lambda_{2}, \lambda_{1} \lambda_{2}\right)$.

A well-known theorem of L. Lempert states that on convex domains Carathéodory and Kobayashi pseudodistances coincide (see [4], and also $[3,2])$. It turns out that the same is true on $\mathbb{G}_{2}$. So, it is important to know whether $\mathbb{G}_{2}$ can be presented as an exhaustion of domains biholomorphic to convex domains.

In [1] C. Costara proved that $\mathbb{G}_{2}$ is not biholomorphic to a convex domain. Using similar arguments we show the following improvement.

TheOREM 1. $\mathbb{G}_{2}$ cannot be exhausted by domains biholomorphic to convex domains.

Proof. Note that $\pi$ is a proper holomorphic mapping. Let $\varrho(s, p)=$ $\max \left\{\left|\lambda_{1}\right|,\left|\lambda_{2}\right|\right\}$, where $\lambda_{1}, \lambda_{2}$ are such that $\pi\left(\lambda_{1}, \lambda_{2}\right)=(s, p)$. It is easy to see that $\varrho$ is a continuous plurisubharmonic function in $\mathbb{C}^{2}$. Moreover, $\varrho\left(\lambda s, \lambda^{2} p\right)=|\lambda| \varrho(s, p)$ for any $\lambda \in \mathbb{C}$ and any $(s, p) \in \mathbb{C}^{2}$. We put $\varphi_{\lambda}\left(z_{1}, z_{2}\right)$ $=\left(\lambda z_{1}, \lambda^{2} z_{2}\right)$. Then $\varrho\left(\varphi_{\lambda}(z)\right)=|\lambda| \varrho(z)$.

One can check that $\mathbb{G}_{2}=\left\{(s, p) \in \mathbb{C}^{2}: \varrho(s, p)<1\right\}$. For any $\varepsilon>0$ we put $G_{\varepsilon}:=\left\{(s, p) \in \mathbb{C}^{2}: \varrho(s, p)<1-\varepsilon\right\}$.

Assume that $U_{\varepsilon}$ is a neighborhood of $\bar{G}_{\varepsilon}$ and $f_{\varepsilon}: U_{\varepsilon} \rightarrow V_{\varepsilon}$ is a biholomorphic mapping, where $V_{\varepsilon}$ is a convex domain. We may assume that $f_{\varepsilon}(0)=0$ and that $f_{\varepsilon}^{\prime}(0)=$ id $($ see $[1])$.

2000 Mathematics Subject Classification: Primary 32H35.

Key words and phrases: symmetrized bidisc.

The author was supported in part by the KBN grant No. 5 P03A 03321. 
Fix $\left(s_{1}, p_{1}\right),\left(s_{2}, p_{2}\right) \in \mathbb{C}^{2}$ and $r \in[0,1]$. Put

$$
\begin{aligned}
R & :=\max \left\{\varrho\left(s_{1}, p_{1}\right), \varrho\left(s_{2}, p_{2}\right)\right\}, \\
g_{\varepsilon}(\lambda) & :=f_{\varepsilon}^{-1}\left(r f_{\varepsilon}\left(\lambda s_{1}, \lambda^{2} p_{1}\right)+(1-r) f_{\varepsilon}\left(\lambda s_{2}, \lambda^{2} p_{2}\right)\right) .
\end{aligned}
$$

We have $g_{\varepsilon}(0)=0$. Note that $g_{\varepsilon}$ is well defined for $|\lambda|<(1-\varepsilon) / R$. Indeed, $\varrho\left(\varphi_{\lambda}\left(s_{j}, p_{j}\right)\right)=|\lambda| \varrho\left(s_{j}, p_{j}\right) \leq R|\lambda|<1-\varepsilon$ for $j=1,2$. Moreover, we have $\varrho\left(g_{\varepsilon}(\lambda)\right) \leq 1$ for any $|\lambda|<(1-\varepsilon) / R$. Let $h_{\varepsilon}(\lambda)=\varphi_{1 / \lambda}\left(g_{\varepsilon}(\lambda)\right)$. Then $h_{\varepsilon}$ : $\mathbb{D}(0,(1-\varepsilon) / R) \backslash\{0\} \rightarrow \mathbb{C}^{2}$ is a holomorphic mapping. Moreover, it extends holomorphically to 0 . Set $g_{\varepsilon}=\left(\left(g_{\varepsilon}\right)_{1},\left(g_{\varepsilon}\right)_{2}\right)$. Simple calculations show

(1) $\left(\left(g_{\varepsilon}\right)_{1}\right)^{\prime}(0)=r s_{1}+(1-r) s_{2}$;

(2) $\left(\left(g_{\varepsilon}\right)_{2}\right)^{\prime}(0)=0$

(3) $\left(\left(g_{\varepsilon}\right)_{2}\right)^{\prime \prime}(0)=2\left(r p_{1}+(1-r) p_{2}\right)$

$$
+\frac{\partial^{2}\left(\left(f_{\varepsilon}\right)_{2}\right)}{\partial s^{2}}(0)\left(r s_{1}^{2}+(1-r) s_{2}^{2}-\left(r s_{1}+(1-r) s_{2}\right)^{2}\right) \text {. }
$$

Put

$$
t_{\varepsilon}=\frac{1}{2} \cdot \frac{\partial^{2}\left(\left(f_{\varepsilon}\right)_{2}\right)}{\partial s^{2}}(0)
$$

Then

$$
h_{\varepsilon}(0)=\left(r s_{1}+(1-r) s_{2}, r p_{1}+(1-r) p_{2}+t_{\varepsilon} r(1-r)\left(s_{1}-s_{2}\right)^{2}\right) .
$$

By the maximum principle $\varrho\left(h_{\varepsilon}(\lambda)\right) \leq \max _{|\mu|=t} \varrho\left(h_{\varepsilon}(\mu)\right)$. But for $\lambda \neq 0$ we have

$$
\varrho\left(h_{\varepsilon}(\lambda)\right)=\varrho\left(\varphi_{1 / \lambda}\left(g_{\varepsilon}(\lambda)\right)\right)=\frac{1}{|\lambda|} \varrho\left(g_{\varepsilon}(\lambda)\right) \leq \frac{1}{|\lambda|} .
$$

Hence,

$$
\varrho\left(h_{\varepsilon}(0)\right) \leq \frac{R}{1-\varepsilon} .
$$

Write $t_{\varepsilon}=e^{i \theta}\left|t_{\varepsilon}\right|$. Take $r=1 / 2,\left(s_{1}, p_{1}\right)=\pi(\zeta,-1)$, and $\left(s_{2}, p_{2}\right)=\pi(\zeta, 1)$, where $\zeta=e^{i(\theta+\pi) / 2}$. Note that $t_{\varepsilon}=-\zeta^{2}\left|t_{\varepsilon}\right|$. We have $\varrho\left(1,-\left|t_{\varepsilon}\right|\right)=\varrho\left(\zeta, t_{\varepsilon}\right) \leq$ $1 /(1-\varepsilon)$. From this we get

$$
\frac{1+\sqrt{1+4\left|t_{\varepsilon}\right|}}{2}=\varrho\left(1,-\left|t_{\varepsilon}\right|\right) \leq \frac{1}{1-\varepsilon} .
$$

So, $t_{\varepsilon} \rightarrow 0$ as $\varepsilon \rightarrow 0$. Letting $\varepsilon \rightarrow 0$ in (1) we get

$$
\varrho\left(r s_{1}+(1-r) s_{2}, r p_{1}+(1-r) p_{2}\right) \leq \max \left\{\varrho\left(s_{1}, p_{1}\right), \varrho\left(s_{2}, p_{2}\right)\right\},
$$

which contradicts the non-convexity of $\mathbb{G}_{2}$.

\section{References}

[1] C. Costara, The symmetrized bidisc as a counterexample to the converse of Lempert's theorem, Bull. London Math. Soc., to appear (2003). 
[2] A. Edigarian, A remark on the Lempert theorem, Univ. Iag. Acta Math. 32 (1995), 83-86.

[3] M. Jarnicki and P. Pflug, Invariant Distances and Metrics in Complex Analysis, de Gruyter, 1993.

[4] L. Lempert, La métrique de Kobayashi et la représentation des domaines sur la boule, Bull. Soc. Math. France 109 (1981), 427-474.

Institute of Mathematics

Jagiellonian University

Reymonta 4/526

30-059 Kraków, Poland

E-mail: Armen.Edigarian@im.uj.edu.pl 